\title{
BMJ Open Evaluation of the overdiagnosis in breast screening programmes using a Monte Carlo simulation tool: a study of the influence of the parameters defining the programme configuration
}

\author{
Cristina Forastero, ${ }^{1}$ Luis I Zamora, ${ }^{2}$ Damián Guirado, ${ }^{1,3}$ Antonio M Lallena ${ }^{\oplus 4}$
}

To cite: Forastero C, Zamora LI, Guirado D, et al. Evaluation of the overdiagnosis in breast screening programmes using a Monte Carlo simulation tool: a study of the influence of the parameters defining the programme configuration. BMJ Open 2019;9:e023187. doi:10.1136/ bmjopen-2018-023187

- Prepublication history for this paper is available online. To view these files, please visit the journal online (http://dx.doi. org/10.1136/bmjopen-2018023187).

Received 25 March 2018 Revised 20 December 2018 Accepted 8 January 2019

\section{Check for updates}

(C) Author(s) (or their employer(s)) 2019. Re-use permitted under CC BY-NC. No commercial re-use. See rights and permissions. Published by BMJ.

${ }^{1}$ Unidad de Radiofísica, Hospital Univ. San Cecilio, Granada, Spain ${ }^{2}$ Servicio de Física y Protección Radiológica, Hospital Reg. Univ. Virgen de las Nieves, Granada, Spain

${ }^{3}$ CIBER de Epidemiología y Salud Pública (CIBERESP), Granada,

Spain

${ }^{4}$ Departamento de Física Atómica, Molecular y Nuclear, Universidad de Granada, Granada, Spain

Correspondence to Professor Antonio M Lallena; lallena@ugr.es

\section{ABSTRACT}

Objectives To build up and test a Monte Carlo simulation procedure for the investigation of overdiagnosis in breast screening programmes (BSPs).

Design A Monte Carlo tool previously developed has been adapted for obtaining the quantities of interest in order to determine the overdiagnosis: the annual and cumulative number of cancers detected by screening, plus interval cancers, for a population following the BSP, and detected clinically for the same population in the absence of screening. Overdiagnosis is obtained by comparing these results in a direct way.

Results Overdiagnosis between $7 \%$ and $20 \%$, depending on the specific configuration of the programme, have been found. These range of values is in agreement with some of the results available for actual BSPs. In the cases analysed a reduction of $11 \%$ at most has been found in the number of invasive tumours detected by screening in comparison to those clinically detected in the control population. It has been possible to establish that overdiagnosis is almost entirely linked to ductal carcinoma in situ tumours.

Conclusions The use of Monte Carlo tools may facilitate the analysis of overdiagnosis in actual BSPs, permitting to address the role played by various quantities of relevance for them.

\section{INTRODUCTION}

Breast screening programmes (BSPs) have become usual in developed countries and this has stimulated an intense debate about the potential risks and benefits for the women invited to follow them. One of their main consequences is the change of the breast cancer incidence in the population. This is due to the advance in the diagnosis of the disease, which increases the incidence rates at earlier ages. In addition, other facts, such as those derived from the use of hormone replacement therapy, may modify the prevalence and, eventually increase the underlying incidence. $^{1}$
Strengths and limitations of this study

- Overdiagnosis values quoted in the present study may be slightly overestimated because we have chosen 70 years as the maximum age of the women following the breast screening programmes (BSPs).

- Simulations require the use of data corresponding to a reference population (eg, the breast cancer incidence) that may differ to some extent with those of specific populations and that introduces a certain uncertainty in the results.

- The use of the present Monte Carlo tool permits to investigate the influence of the parameters defining the BSP as well as their results (such as overdiganosis) without involving actual populations and avoiding the economical, logistic and ethical problems that it usually entails.

- Specifically, overdiagnosis may be addressed in an easy way overcoming the limitations of the randomized essays in this respect.

- Possible changes in the diagnostic or procedural methodologies can be included in the simulation, thus allowing to anticipate the results of the new BSPs before their implementation.

However, this apparent incidence excess should not be confused with overdiagnosis, an aspect that, together with overtreatment, has focused part of the discussion in the last years. ${ }^{2}$ BSPs are supposed to enhance the ability for detecting tumours in their early growth stages, which are those associated with a better prognosis and survival of patients. However, some of the cancers found in the screening grow so slowly that they would never manifest clinical symptoms before the women will die due to causes other than breast cancer. The detection of these tumours is called overdiagnosis of the BSP. $^{23}$ Due to the impossibility of knowing, a priori, the cancer evolution, the available therapeutic machinery is switched on and the 
women overdiagnosed become subject of overtreatment (eg, surgery, radiotherapy and even chemotherapy) with the consequent possible adverse effects, even in those cases that would not evolve towards malignancy. Besides, some degree of spontaneous remission or asymptomatic latency, in case of invasive cancers, has been observed, and this would increase overdiagnosis. ${ }^{45}$

The determination of the overdiagnosis extent is in practice of a complicated task. Some estimations have been provided on the basis of the results obtained in BSPs running after several years. In this case, overdiagnosis is obtained by comparing the incidence of breast cancer in the women following the BSP with that in a population with similar characteristics (age, exposure to risk factors for breast cancer, availability of treatment, follow-up until death, etc.) and that does not undergo screening. Overdiagnosis would be linked to the cancer excess in the BSP group but the direct interpretation of the incidence data in women following a BSP is not straightforward because of the fluctuations in the incidences of the populations that are compared, the differences in the configurations of the BSPs considered (age ranges of the women following the programme, mammography frequencies, follow-up times), as well as the ways how the lead time (understood as the time between the tumour detection by screening and the clinical one) is considered. ${ }^{26}$

A priori, a most consistent estimate of overdiagnosis may be obtained in combined analyses of the results of various randomised controlled trials (RCTs). However, a huge variability in the quoted overdiagnosis data, mainly due to different methodologies in observational studies, has been pointed out. ${ }^{67}$ In any case, the RCTs developed to date ${ }^{8-15}$ were not designed to determine overdiagnosis and, as most of them began in the 1980s, have not included most of the recent advances in therapy and have not taken into account the impact of the higher image quality and the reduction in the detection thresholds on it.

In these circumstances, Monte Carlo simulation appears to be as a good alternative to overcome the difficulties pointed out. In previous works, ${ }^{16-18}$ we have developed a mammographic screening model based on Monte Carlo techniques and aiming at evaluating screening programmes. This simulation tool has allowed us to reproduce the results of actual BSPs concerning the invasive/in situ tumour detection rates with and without screening, the ranges of interval cancers, and other magnitudes associated with detection. By introducing the survival following local-regional treatment has enabled us to make estimates of the mortality reduction attributable to BSPs. Finally, the internal validity of the main RCTs included in the Cochrane meta-analysis ${ }^{3}$ was analysed by using the Monte Carlo tool adequately adapted to take into account the specific characteristics of each of them. In all the situations studied, the Monte Carlo simulation has proven to be an efficient instrument.

The purpose of the present work is to develop a new Monte Carlo tool to investigate the overdiagnosis of BSPs according to their particular configurations, these are age ranges of the women following the programme and mammography frequencies. To do that we have used the tool previously built up and tested ${ }^{16}$ that involves generic distributions that may be adapted to each specific population in order to reach enough precision in the simulation. The programme will provide us with the annual and cumulative number of cancers detected by screening, as well as the number of cancers clinically detected during the programme running (interval cancers) of the population undergoing screening. The comparison of these results with those obtained for the same population in the absence of screening has allowed us to estimate the overdiagnosis in a direct way.

\section{MATERIAL AND METHODS}

Our Monte Carlo tool allows carrying out simulations based on a minimum number of parameters having clinical or physical significance: tumour average size for symptomatic detection, mammography sensitivity (as a function of the thickness, histological type and density of the breast) and the proportion of invasive/in situ tumours. It is worth reminding the differences between these two histological types of cancers entering in our analysis. Ductal carcinoma in situ (DCIS), or intraductal carcinoma, involves the clonal proliferation of malignant cells. They grow in the mammary duct lumens, do not invade the adjacent breast stroma and are usually diagnosed by mammography. On the contrary, invasive tumours do multiply in normal tissues and constitute much of the cases of the clinically detected breast cancers. Besides, we do not include any ad-hoc assumption such as that related to the higher aggressivity of tumours in their early growth stages.

Other simulation models are either overparameterised or based on distributions of parameters that are difficult to observe or show non-negligible correlations with the mammography sensitivity. ${ }^{19}$ Other approaches, such as those linked to Markov models, may generate results that strongly depend on the defined states. ${ }^{20}$

In the simulation tool we developed, ${ }^{16}$ that is the base of the present Monte Carlo approach, the history of each woman participating in a BSP is simulated individually, considering a model that includes parameters and characteristics that may be gathered in one of the following categories.

- Parameters describing the target population of the BSP. Here we have the incidence distribution of breast cancer as a function of the age of the women. In our simulations we have used that of the Canary Islands before BSP as representative of an occidental population not submitted to screening. As the incidence actually needed is that of the tumour onset, the incidence curve was shifted towards smaller ages using the average time between the tumour detection by any diagnostic technique and the tumour onset, employing a logistic growth model. 
- Tumour properties that influence the probability of detection by mammography. Tumour size, histological type and breast density are the three fundamental characteristics in this group. In addition, a model describing the evolution of the tumour size as a function of time and a model of the detection probability of breast cancer by mammography are required.

- Configuration of the BSP. Here we included the frequency of mammography, the age range of the women participating in the programme and the follow-up time for each participant.

The simulation of a history begins by sampling, within the corresponding population distribution, the age of a candidate to participate in the BSP. If the age obtained is within the range established for the virtual BSP, the women history continues by sampling whether the woman has developed a cancer at some moment since her birth. This is done according to the cumulative incidence distribution. If the answer is affirmative, then the women breast density, the initial histological type of the tumour, the moment when it occurred and its size when the woman enters into the BSP are obtained by sampling the corresponding distributions. It is worth pointing out that both the tumour histological type and the breast density may change throughout the simulated history. The histological type may evolve from DCIS to invasive cancers. Also the breast density may reduce with age. These transitions are hypotheses taken into account in our model.

The moment at which the tumour arises is sampled according to the incidence curve, which shows the probability of tumour occurrence as a function of the woman age. The tumour size is estimated by using a realistic tumour growth model that describes the increase of the tumour diameter with the time elapsed since its inception. This growth is simulated by using a model ${ }^{21}$ with a single free parameter that we assume to follow a log-normal distribution, an initial proportion of invasive/ DCIS tumours, and given transition probabilities between these two tumour histological types. This proportion is chosen according the results obtained for detection in the various screening rounds. No additional hypotheses about tumour evolution are included.

In those cases in which women have developed a tumour, the detection probability is sampled to consider whether the tumour is detected or not after the mammography. For women who do not present disease, the specificity is used. If a true positive case occurs, the history is finished and a new one is started. In any other case, successive rounds are simulated either because the tumour was not detected in the previous one (false negative) or the woman is healthy but continues in the BSP until the age limit is reached (true negative). If, in this last case, the woman was erroneously classified as positive, we would deal with a false positive case and it is understood that the woman is called again for a further test that will confirm the error in the diagnosis and that she will continue within the BSP.
In false negative cases, which suppose the continuation of the history, the simulation takes into account the growth of the tumour between the different rounds, including the probability of clinical detection between them. This means that interval cancers are considered. Also the possible changes in the tumour histological type and breast density are taken into account. Likewise, for the cases of healthy women, the probability of cancer occurrence during follow-up is simulated.

With this methodology, the Monte Carlo tool permits to simulate the introduction of a BSP in a given population, carry out its follow-up during a certain time and determine with the selected periodicity the total number of tumours detected by screening in the sample of women that attend the programme, as well as those detected clinically (interval cancers if they occur in women who follow the BSP). If the prevalence of breast cancer is stable over time, the incidence curves corresponding to the population undergoing screening should converge, after a time of the order of the lead time, to those found for the same population without screening, and the whole procedure would not show overdiagnosis. If this does not happen, and after the lead time, the two cumulative incidence curves would remain parallel and the difference between both would provide us with an estimate of the overdiagnosis.

Specifically, the overdiagnosis was calculated $\mathrm{as}^{2}$ :

$$
S=\frac{N_{\text {screen }}\left(t_{\mathrm{c}}+t_{1}\right)-N_{\text {clin }}\left(t_{\mathrm{c}}+t_{1}\right)}{N_{\text {screen }}\left(t_{\mathrm{c}}\right)}
$$

where $N_{\text {screen }}(t)$ is the cumulative number of cancers detected in women who have followed the BSP during a total time $t$, including interval cancers, $N_{\text {clin }}(t)$ is the cumulative number of tumour detected clinically in an equivalent group of women who have not followed a BSP, $t_{\mathrm{C}}$ is the duration of the BSP or time course and $t_{1}$ is a time required to avoid the transient period associated to the lead time. Except for a very small number of cancers, the average lead time is $\sim 3-4$ years $^{2}$ and we have considered $t_{1}=10$ years.

In our simulations we ran 50 million of woman histories in each BSP. Two age ranges $\left[a_{1}, a_{\mathrm{u}}\right]=[50,70]$ and $[40,70]$ (with age values given in years) were analysed with $t_{\mathrm{c}}=10$ and 20 years, respectively. Time intervals between consecutive mammographies of $t_{\text {int }}=1,2$ and 3 years were considered. In all cases simulated, an attendance rate of $100 \%$ was assumed, though estimations for $80 \%$ attendance were also performed.

The first running year of the BSP, all women with ages in the selected age range $\left[a_{1}, a_{\mathrm{u}}\right]$ enter the programme; in the following years, women who reach the minimum age of participation $a_{1}$ are incorporated. They are followed within the BSP until they reach $a_{\mathrm{u}}$. If the programme finishes, woman's histories continue to be simulated, considering the possibility of tumour clinical detection. In our simulations we chose 20 years for this period after the programme: this permitted estimating the time necessary for the programme effects to disappear. 

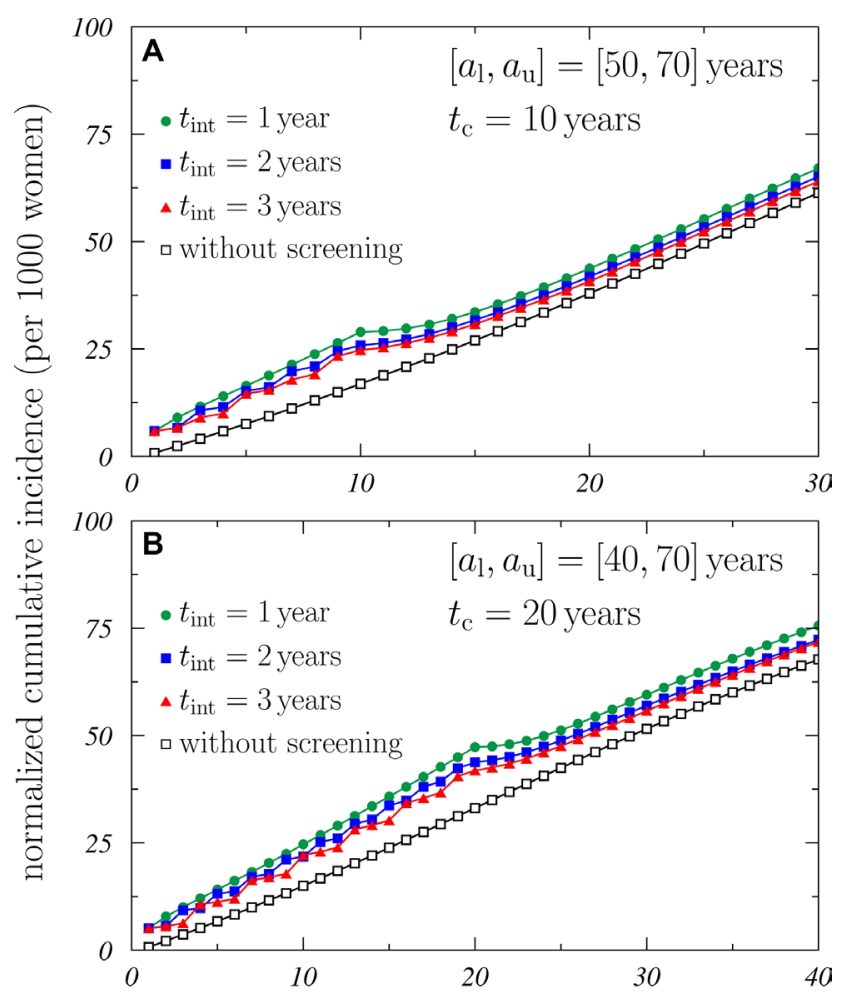

years since the beginning of the BSP

Figure 1 Cumulative invasive + ductal carcinoma in situ tumour incidence, normalised to the average number of women participating in the breast screening programme (BSP), as a function of the time elapsed since the beginning of the BSP. Solid symbols show the results obtained for the configurations $(A)$ [50-70] with a BSP duration of $t_{\mathrm{C}}=10$ years and (B) [40-70] with $t_{\mathrm{C}}=20$ years, with $100 \%$ attendance. The values corresponding to intervals between mammographies of $t_{\text {int }}=1,2$ and 3 years are shown with green solid circles, blue solid squares and red solid triangles, respectively. Open squares indicate the results found for the woman population who do not follow the BSP.

\section{Patient and public involvement}

The present study deals with a Monte Carlo simulation. Then, neither patients nor public were involved.

\section{RESULTS}

Figure 1 shows the cumulative incidences for invasive plus DCIS tumour, normalized to the average number of women participating in the BSP, (per thousand women), as a function of the time elapsed since the beginning of the BSP, for the configurations of [50-70] with $t_{\mathrm{c}}=10$ years (panel 1a) and [40-70] with $t_{\mathrm{C}}=20$ years (panel $1 b)$. For these configurations, the results obtained for mammography frequencies $t_{\text {int }}=1$ (green solid circles), 2 (blue solid squares) and 3 (red solid triangles) years are shown. The attendance is assumed to be $100 \%$. Open squares correspond to the values found for the population of women who do not followed the BSP.

The overdiagnosis estimates for $100 \%$ attendance, calculated according to equation (1), are shown in table 1.

The corresponding incidences for invasive plus DCIS tumours, normalised to the average number of women participating in the BSP and for $100 \%$ attendance, are shown in figure 2.

Finally, in figure 3 the cumulative incidence for invasive cancers in the screened woman sample for $t_{\text {int }}=1$ year and $100 \%$ attendance (green solid circles) is compared to the results obtained for a population of women who did not follow a BSP (open squares). The values are normalised to the average number of women participating in the BSP.

\section{DISCUSSION}

The effect of the overdiagnosis is clearly seen in figure 1 : once a time equal to the lead time $t_{1}=10$ years has passed after the BSP finished (this means, respectively, 20 and 30 years after the beginning of the programme for the two configurations studied), the results obtained in the case of the women who have followed the BSP behave parallel to those of the unscreened woman.

As shown in table 1, the corresponding overdiagnosis estimates found for the [50-70] configuration are significantly larger (between $15 \%$ and $21 \%$ ) than those obtained for the [40-70] one. This is due to the fact that the tumour detection by screening at earlier ages, as it occurs in the second configuration, would have more time to grow and reach a size exceeding the clinical detection threshold in the absence of screening.

On the other hand, and as it was expected, the increase of the time interval between mammographies reduces the overdiagnosis, the reduction being $\sim 40 \%$ when $t_{\text {int }}$ changes from 1 to 3 years.

Table 1 Values of overdiagnosis, as defined in equation (1), for the two configurations studied and the three mammography intervals $t_{\text {int }}$ considered in each case

\begin{tabular}{|c|c|c|c|c|}
\hline \multirow[b]{3}{*}{$t_{\text {int }}$} & \multicolumn{4}{|l|}{ Overdiagnosis } \\
\hline & \multicolumn{2}{|c|}{$[50,70]$ years; $t_{\mathrm{C}}=10$ years } & \multicolumn{2}{|c|}{$[40,70]$ years; $t_{\mathrm{C}}=20$ years } \\
\hline & $100 \%$ attendance & $80 \%$ attendance & $100 \%$ attendance & $80 \%$ attendance \\
\hline 1 year & $0.200 \pm 0.009$ & 0.14 & $0.168 \pm 0.005$ & 0.13 \\
\hline 2 years & $0.150 \pm 0.010$ & 0.10 & $0.123 \pm 0.005$ & 0.07 \\
\hline 3 years & $0.115 \pm 0.007$ & 0.06 & $0.100 \pm 0.005$ & 0.07 \\
\hline
\end{tabular}

Values shown for $100 \%$ attendance were obtained with the Monte Carlo simulation tool; those for $80 \%$ attendance are estimations (see text for details). Uncertainties are given with a coverage factor $k=3$. 


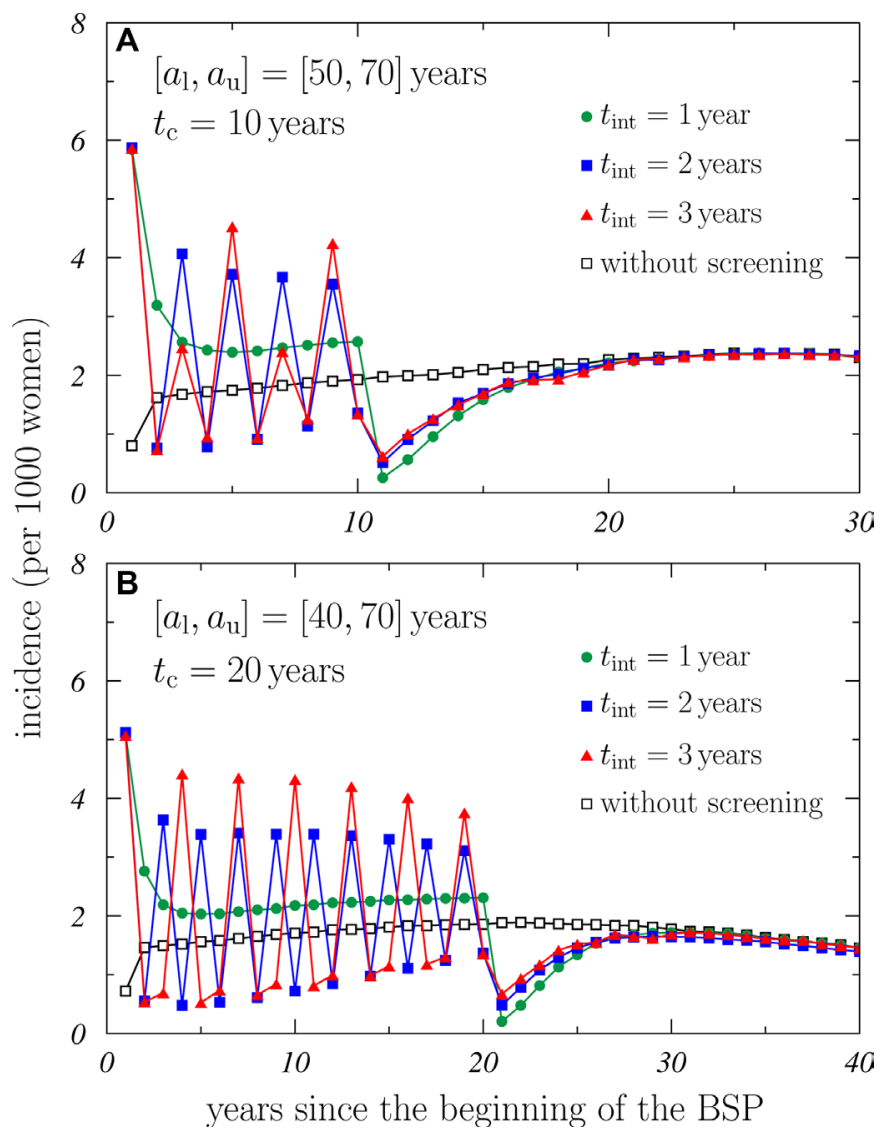

Figure 2 Invasive+ductal carcinoma in situ tumour incidence, normalised to the average number of women participating in the breast screening programme (BSP), as a function of the time elapsed since the beginning of the BSP. Solid symbols show the results obtained for the configurations (A) [50-70] with a BSP duration of $t_{\mathrm{C}}=10$ years and $(B)$ [40-70] with $t_{C}=20$ years, with $100 \%$ attendance. The values corresponding to intervals between mammographies of $t_{\text {int }}=1,2$ and 3 are shown with green solid circles, blue solid squares and red solid triangles, respectively. Open squares indicate the results found for the woman population who do not follow the BSP.

When the BSP finishes (after 10 years in the case of the configuration [50-70] and 20 years for the [40-70] one of its beginning), a transient period occurs. In figure 1 this is better seen in the case of $t_{\text {int }}=1$ year (green solid circles) and it shows up as a change in the slope of the corresponding data. This behaviour may be observed in a more clear way by looking at the corresponding incidences, shown in figure 2. Therein the trend described appears as a strong reduction of the incidence rate and is a consequence of the detection deficit once the BSP is no longer active. As we can see, the incidence rate requires about 10 years to reach the incidence corresponding to the unscreened population (shown with open squares). It is worth mentioning that the reduction observed is inherent to the BSP itself and it occurs even if overdiagnosis is absent. To isolate in the incidence curves these changes from those due to overdiagnosis, it is mandatory waiting for the lead time $t_{1}=10$ years after the BSP has finished.
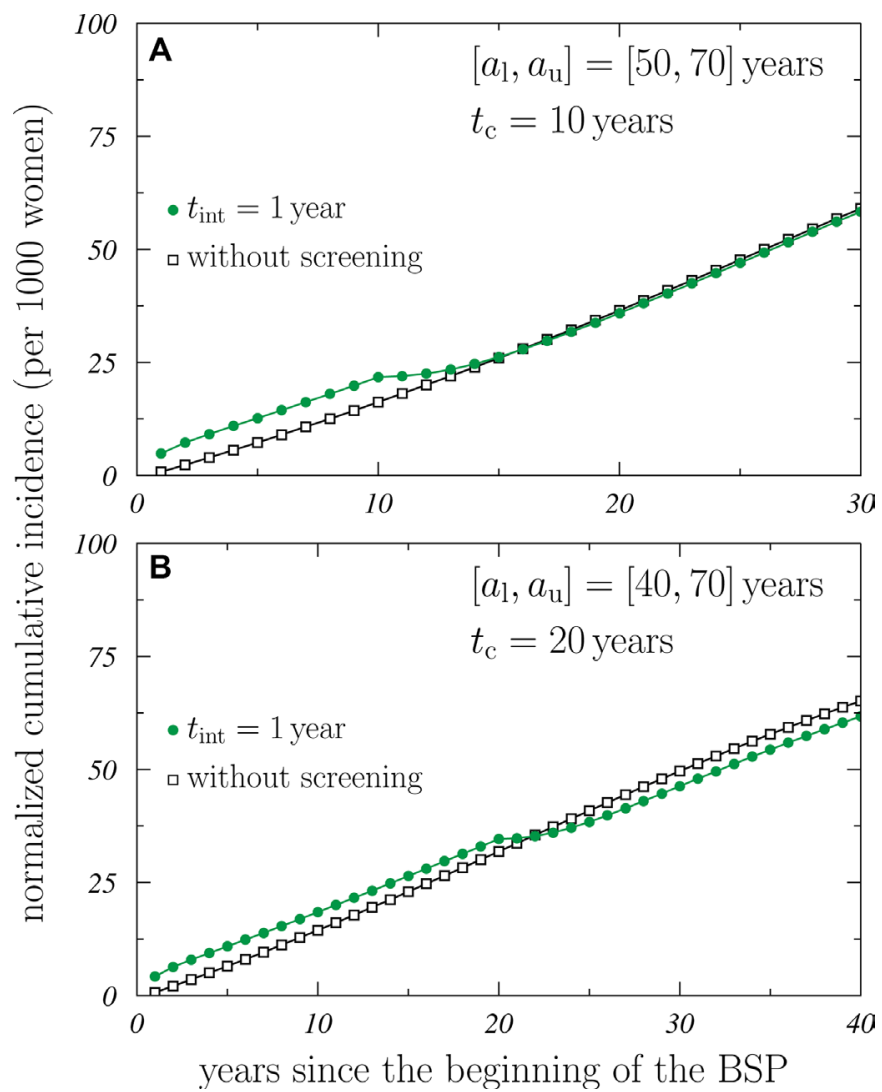

Figure 3 Cumulative incidence for invasive tumours, normalised to the average number of women participating in the breast screening programme (BSP), as a function of the time elapsed since the beginning of the BSP. Green solid circles show the results obtained for the configurations $(A)$ [50-70] with a BSP duration of $t_{\mathrm{C}}=10$ years and (B) [40-70] with $t_{\mathrm{C}}=20$ years, with an interval between mammographies of $t_{\text {int }}=1$ year and $100 \%$ attendance. Open squares indicate the results found for the woman population who do not follow the BSP.

As seen in figure 3, for the configuration [50-70], the cumulative incidence for invasive cancers in the screened woman sample (shown by green solid circles in the panel 3a) coincide with that obtained for the reference population of women not following a BSP (open squares) after a period of $\sim 5$ years once the programme has finished. As the number of invasive tumours is the same in the populations with and without screening, the overdiagnosis above discussed relies completely on DCIS tumours. The same does not occur in the case of the configuration [40-70] (panel 3b) where a deficit of $11 \%$ in the cumulative incidence of invasive tumours with respect to the unscreened population is found after BSP finished.

Once the importance of DCIS tumours in overdiagnosis has been pointed out, it is worth noting that it is not a consequence of a slower growth of these tumours compared with invasive ones, since our model does not include any difference according to their histological type. The reason is rather that there is a greater abundance of DCIS cancers in the early stages in which they can be efficiently detected by screening. 
As said above, overdiagnosis estimations from actual BSPs are difficult. Puliti et $a t^{22}$ analysed 13 observational studies and concluded that overdiagnosis would reach up to $10 \%$. In the case of the British National Health System BSP, with a follow-up of 20 years and for women older than 50 years, ${ }^{2}$ the mammographic screening avoided a death each 250 invited women. Therefore, assuming an acceptance/participation rate of around $80 \%$ and a detection rate in the incidence rounds of 4 cancers detected per 1000 women and year, one would have 3 cases of overdiagnosis/overtreatment per death avoided, that is, $1 \%$ of women invited to BSP are expected to be overdiagnosed.

The situation of the RCT estimations is also unclear. The Cochrane report established an absolute risk of overdiagnosis/overtreatment of $0.5 \% .^{3}$ Much smaller values, $0.025 \%$ per year, were established by Moss $^{23}$ according again to the data of the Canadian trials, ${ }^{910}$ while for the relative risk in the intervention arms of these RCTs he found values between $11 \%$ and $14 \%$. The reasons of these discrepancies may be linked to the serious methodological problems shown by these RCTs. ${ }^{24}$

On the other hand, the 2009 revision for the US Preventive Task Force ${ }^{25}$ quoted that overdiagnosis was within $1 \%$ and $10 \%$.

Testing the results of these actual BSPs or RCTs with our Monte Carlo tool would require detailed simulations in which the various specifications of each of them are included. In the case of BSPs, one of the main inputs is the attendance that in our calculations has been assumed to be $100 \%$. However, it is possible to estimate the overdiagnosis for lower attendances using the results we have obtained by assuming that the number of detected tumours remains approximately constant after the screening has finished. In table 1 the values obtained in this way for $80 \%$ attendance are shown. A smaller attendance give rise to lower values of the overdiagnosis: a reduction of $\sim 5 \%$ has been found for $80 \%$ attendance with respect to $100 \%$ one, the overdiagnosis ranging between $7 \%$ and $14 \%$ depending on the configuration. These estimations give an idea of the magnitude of overdiagnosis in BSPs with attendances around $80 \%$.

To finish, it should be noted that our simulations may be affected by some limitations because we have not the incidence curves corrected by the average detection time for women over 70 years and the simulated histories stopped at that age. This may imply a small overestimation of overdiagnosis. On the other hand, the fact that the simulations carried out require information corresponding to a reference population (eg, the breast cancer incidence) introduces a certain uncertainty in the results because these data may differ to some extent with those of the specific populations investigated.

\section{CONCLUSIONS}

We have developed a model based on Monte Carlo simulation techniques that allows us to reproduce consistently the known results about overdiagnosis on BSPs. It varies from $7 \%$ to $20 \%$ depending the programme configuration (age range of women involved and frequency of mammography).

It has been found that, after the end of the screening programme, the incidence of invasive cancers is similar to that found for an unscreened control group: this implies that overdiagnosis is mainly associated with DCIS tumours. This is due to the fact that this type of cancers are more abundant than the invasive ones when they reach the size that make them to be detectable by mammography.

In any case, Monte Carlo tools appear to be very helpful for analysing the role of the various parameters defining the BSP configurations as well as their results (such as overdiagnosis) without involving actual populations and avoiding the economical, logistic and ethical problems that it usually entails. Even more, changes in the diagnostic or procedural methodologies can be included in the simulation, thus allowing to anticipate the results of the new programmes before their implementation.

Contributors All the authors contributed to the conception and design of the study. CF and LIZ wrote the simulation codes and ran them for the various configurations analysed. All the authors performed the analysis of the simulation results, contributed to the writing and editing of the original version of the manuscript, responded to the reviewers' reports and wrote the revised version of the article.

Funding Work partially supported by the Biomedical Research Networking CenterCIBER de Epidemiología y Salud Pública (CIBERESP), the Spanish Ministerio de Ciencia y Competitividad (FPA2015-67694), the European Regional Development Fund (ERDF) and the Junta de Andalucía (FQM0387).

Competing interests None declared.

Patient consent for publication Not required.

Provenance and peer review Not commissioned; externally peer reviewed.

Data sharing statement There are not additional data from the present study to be shared. Information about the details of the simulations can be obtained from any of the authors.

Open access This is an open access article distributed in accordance with the Creative Commons Attribution Non Commercial (CC BY-NC 4.0) license, which permits others to distribute, remix, adapt, build upon this work non-commercially, and license their derivative works on different terms, provided the original work is properly cited, appropriate credit is given, any changes made indicated, and the use is non-commercial. See: http://creativecommons.org/licenses/by-nc/4.0/.

\section{REFERENCES}

1. Duffy SW, Lynge $\mathrm{E}$, Jonsson $\mathrm{H}$, et al. Complexities in the estimation of overdiagnosis in breast cancer screening. $\mathrm{Br} J$ Cancer 2008;99:1176-8.

2. Independent UK Panel on Breast Cancer Screening. The benefits and harms of breast cancer screening: an independent review. Lancet 2012;380:1778-86.

3. Gøtzsche PC, Nielssen M. Screening for brest cancer with mammography (Review). The Cochrane Collaboration. Copenhagen: John Wiley \& Sons, 2009.

4. Zahl PH, Maehlen J, Welch HG. The natural history of invasive breast cancers detected by screening mammography. Arch Intern Med 2008;168:2311-6.

5. Zahl PH, Gøtzsche PC, Mæhlen J. Natural history of breast cancers detected in the Swedish mammography screening programme: a cohort study. Lancet Oncol 2011;12:1118-24.

6. Biesheuvel C, Barratt A, Howard K, et al. Effects of study methods and biases on estimates of invasive breast cancer overdetection with mammography screening: a systematic review. Lancet Oncol 2007;8:1129-38.

7. Puliti D, Miccinesi G, Paci E. Overdiagnosis in breast cancer: design and methods of estimation in observational studies. Prev Med 2011;53:131-3. 
8. Andersson I, Aspegren K, Janzon L, et al. Mammographic screening and mortality from breast cancer: the Malmö mammographic screening trial. BMJ 1988;297:943-8.

9. Miller AB, To T, Baines CJ, et al. Canadian National Breast Screening Study-2: 13-year results of a randomized trial in women aged 50-59 years. J Natl Cancer Inst 2000;92:1490-9.

10. Miller AB, To T, Baines CJ, et al. The Canadian National Breast Screening Study-1: breast cancer mortality after 11 to 16 years of follow-up. A randomized screening trial of mammography in women age 40 to 49 years. Ann Intern Med 2002;137:305-12.

11. Tabàr L, Gad A. Screening for breast cancer: the Swedish trial. Radiology 1981;138:219-22.

12. Shapiro S, Venet W, Strax P, et al. Selection, follow-up, and analysis in the Health Insurance Plan Study: a randomized trial with breast cancer screening. Natl Cancer Inst Monogr 1985;67:65-74.

13. Frisell J, Glas U, Hellström L, et al. Randomized mammographic screening for breast cancer in Stockholm. Design, first round results and comparisons. Breast Cancer Res Treat 1986;8:45-54.

14. Bjurstam N, Björneld L, Duffy SW, et al. The Gothenburg breast screening trial: first results on mortality, incidence, and mode of detection for women ages 39-49 years at randomization. Cancer 1997;80:2091-9.

15. Moss SM, Wale C, Smith R, et al. Effect of mammographic screening from age 40 years on breast cancer mortality in the UK Age tria at 17 years' follow-up: a randomised controlled trial. Lancet Oncol 2015;16:1123-32.

16. Forastero C, Zamora LI, Guirado D, et al. A Monte Carlo tool to simulate breast cancer screening programmes. Phys Med Biol 2010;55:5213-29.
17. Zamora LI, Forastero C, Guirado D, et al. A Monte Carlo tool to study the mortality reduction due to breast screening programs. Med Phys 2012;39:7215-23.

18. Zamora LI, Forastero C, Guirado D, et al. A Monte Carlo analysis of breast screening randomized trials. Phys Med 2016;32:1609-14.

19. Cong XJ, Shen Y, Miller AB. Estimation of age-specific sensitivity and sojourn time in breast cancer screening studies. Stat Med 2005;24:3123-38.

20. Uhry Z, Hédelin G, Colonna M, et al. Multi-state Markov models in cancer screening evaluation: a brief review and case study. Stat Methods Med Res 2010;19:463-86.

21. Spratt JA, von Fournier D, Spratt JS, et al. Decelerating growth and human breast cancer. Cancer 1993;71:2013-9.

22. Puliti D, Duffy SW, Miccinesi G, et al. Overdiagnosis in mammographic screening for breast cancer in Europe: a literature review. J Med Screen 2012;19:42-56.

23. Moss S. Overdiagnosis and overtreatment of breast cancer: overdiagnosis in randomised controlled trials of breast cancer screening. Breast Cancer Res 2005;7:230-4.

24. Njor SH, Garne JP, Lynge E. Over-diagnosis estimate from the Independent UK Panel on Breast Cancer Screening is based on unsuitable data. J Med Screen 2013;20:104-5.

25. Nelson HD, Tyne K, Naik A, et al. Screening for breast cancer: systematic evidence review update for the U. S. Preventive Task Force. Evidence review update No. 74. AHRQ publication No. 10 05142-EF-1. Rockville: Agency for Healthcare Researc and Quality, 2009. 